\title{
Nosocomial Blood Stream Infections in Pediatric Intensive Care Unit of Fauji Foundation Hospital, Rawalpindi Pakistan
}

\author{
Iqra Ayaz ${ }^{1}$, Hira Hameed ${ }^{1}$, Wajeeha Amber ${ }^{1}$, Talal Zafar ${ }^{2}$ \\ ${ }^{1}$ Senior Registrar, Department of Pediatrics, Islamabad Medical and Dental College, Islamabad Pakistan \\ ${ }^{2}$ Senior Registrar, Department of Pediatrics, HBS Medical and Dental College, Islamabad Pakistan
}

\begin{abstract}
Background: Nosocomial blood stream infections with bacterial organisms are one of the most common problems faced by health care professionals in admitted patients, since these infections complicate the treatment and affect the outcome. The objective of this study was to determine the frequency of most common bacterial organisms in nosocomial blood stream infections in children admitted in Pediatric Intensive Care Unit of a tertiary care hospital at Rawalpindi, Pakistan.

Material and Methods: This cross-sectional study was carried out from 6th July 2017 to 6th January 2018 in the Pediatric Intensive Care Unit (ICU), Fauji Foundation Hospital, Rawalpindi, a tertiary care hospital. A total of 385 patients, aged 14 days to 12 years, admitted in the ICU were monitored from the time of admission till their blood culture reports were positive for bacterial growth along with signs of clinical sepsis Blood samples for culture were collected aseptically and the bottles were incubated for 7 days. Patients were monitored from time the blood culture yielded growth of bacterial pathogens till final blood culture report with antibiotics sensitivity against the pathogen became available. Data was analyzed using SPSS Version 20. Effect modifiers like age, duration of admission and gender were controlled by stratification and chi-square test was applied for comparison with $P$-value less than .05 considered as statistically significant.

Results: Pseudomonas aeruginosa and Salmonella typhi were the most common bacterial organisms ( $n=83 ; 21.6 \%$ each) causing nosocomial blood stream infection, followed by Escherichia coli $(n=77 ; 20 \%)$ and Klebsiella ( $n=67 ; 17.4 \%)$, respectively. Effect modifier, like gender (stratified and compared by Chi-square test) had a statistically significant relationship with frequency of most common bacterial organisms in nosocomial blood stream infections $(P=.001)$. However, effect modifiers like age and duration (hours) of admission to PICU had a non-significant relationship with frequency of bacterial organisms in nosocomial blood stream infections $(P>.05)$.

Conclusions: There is a high frequency of common bacterial organisms in nosocomial blood stream infections in children with predominance of gram-negative bacteria including Pseudomonas aeruginosa and Salmonella typhi. Thus, early initiation of appropriate antibiotic therapy can help in decreasing mortality, significantly in hospitalized patients.

Key words: Children, Gram-negative bacteria, Intensive care unit, Nosocomial blood stream infections

Authors' Contribution:
${ }^{1}$ Conception; Literature research;
manuscript design and drafting; ${ }^{2,3}$ Critical
analysis and manuscript review; Data
analysis; Manuscript Editing.

Article info:

Received: April 16, 2020

Accepted: September 25, 2020
\end{abstract}

Cite this article. Ayaz I, Hameed H, Amber W, Zafar T. Nosocomial Blood Stream Infections in Pediatric Intensive Care Unit of Fauji Foundation Hospital, Rawalpindi Pakistan. J Islamabad Med Dental Coll. 2020; 9(4): 269-274. Doi: 10.35787/jimdc.v9i4.533

\author{
Correspondence: \\ Hira Hameed \\ Email: hira.hameed@imdcollege.edu.pk
}

Funding Source: Nil
Conflict of Interest: Nil 


\section{Introduction}

Nosocomial bloodstream infections (nBSIs) are an important cause of morbidity and mortality and are the most frequent type of infections in pediatric patients. The prevalence of health care associated infections is $4.2 \% .{ }^{1}$ Most common type of infections in children are blood stream infections (45\%). Other common infections include lower respiratory tract infections (22\%), gastrointestinal infections (8\%), eye, ear, nose, and throat infections (7\%), urinary tract infections (5\%), and surgical site infections (4\%), respectively. ${ }^{1}$ Among admitted patients, there is highest prevalence of infections in pediatric (PICU) and neonatal intensive care units (NICU) (15.5\% and $10.7 \%$ respectively). ${ }^{1}$ Nosocomial BSIs in PICU are a common but preventable complication in critically ill patients and has a negative impact on patients' outcome ${ }^{2}$. Most of the common bacterial organisms causing nBSIs show a high resistance to commonly used antibiotics including third generation cephalosporins. ${ }^{2}$ ICU acquired infections increase mortality and duration of hospital stay. ${ }^{2}$

There are multiple studies on frequency of BSI, their etiology, common pathogens involved and recommended treatment guidelines. ${ }^{3-5}$ A study by Mitt et al. in January 2004 to December 2008 in mixed pediatric population showed very high rates of blood stream infections. Most common pathogen were coagulase-negative staphylococci (43\%) and Serratia marcescens (14\%). ${ }^{4}$ A multicenter study was done in Spanish PICU in year 2008 to 2012, which concluded that common nBSI were central lineassociated nosocomial infections, ventilator associated pneumonia and catheter associated urinary tract infections. Common microorganisms involved were gram-negative organisms, grampositive organisms and candida species. ${ }^{5}$

A study was conducted to determine the impact of an extended infection control training program in
University Malaya Medical Centre, Malaysia which concluded that education of medical interns on infection control, a relatively low-cost intervention, resulted in a substantial reduction in the incidence of PICU-acquired BSIs. ${ }^{6}$

A study done in Karachi, Pakistan to determine frequency of $\mathrm{BSI}$ in adult population with febrile neutropenia showed that frequency of Staphylococcus aureus was $16 \%$, E. coli $14.5 \%$, Pseudomonas 8.5\% and Klebsiella 15.5 \%. ${ }^{7}$ Another study done at Agha Khan University Hospital, Karachi from 2013 to 2017 concluded that most common pathogen of BSI is staphylococcus non-aureus species $(33.3 \%)^{8}$ It has been observed that frequency and types of nosocomial infections and pathogens causing BSI show demographical variation. ${ }^{9}$ There is limited date available regarding frequency of common pathogens in nosocomial BSIs in children in Punjab.

The purpose of this study was to determine frequency of most common bacterial organisms in nBSIs in children in a tertiary care hospital of Rawalpindi, so that early initiation of appropriate antibiotic therapy can be implemented, which will contribute significantly towards decrease in mortality rates in our setup.

\section{Material and Methods}

This cross-sectional study was carried out at the Department of Pediatric Medicine, Fauji Foundation Hospital, Rawalpindi Pakistan in pediatric intensive care unit (PICU) over a period of 6 months, from $6^{\text {th }}$ July, 2017 to $6^{\text {th }}$ January, 2018. Sample size was calculated by using WHO calculator. The anticipated population proportion was $9.2 \%$ with level of significance $95 \%$ and absolute precision $2.9 \% .{ }^{10} \mathrm{~A}$ total of 385 patients, aged 14 days to 12 years were enrolled by consecutive non probability sampling. 
The patients enrolled in the study had PICU stay of more than 48 hours and they developed signs of clinical sepsis according to their age. Children less than one year of age with either fever more than $38^{\circ} \mathrm{C}$ or Hypothermia less than $37^{\circ} \mathrm{C}$ were defined as having clinical sepsis.

Children already admitted with nosocomial infections acquired from PICU were excluded. Bloodstream infection that occurred after more than 48 hours of admission to PICU or within 48 hours after discharge was defined as nosocomial infection. Ethical approval was taken from Institutional Ethical Review committee. Informed verbal and written consent were obtained from parents regarding use of data for research and publication.

Specimens were collected according to the protocol of the Microbiology laboratory. Blood samples for culture were collected aseptically from fresh peripheral venous pricks and inoculated into blood culture bottles that were incubated for 7 days. All patients at PICU were monitored from the time of admission till they showed sign of infections, based on standard definitions. A microbiology resident and a nursing staff member were specifically given the task of following up all patients from the time the blood culture yielded growth till the report became available. Data was collected on a predesigned proforma. Confidentiality of patients was maintained during the study.

The data was analyzed using SPSS Version 20. Descriptive statistics were calculated for both qualitative and quantitative variables. Frequencies and percentages were calculated for qualitative variables like gender and bacterial organism. Mean and standard deviation were used for quantitative data like age, duration of admission, blood pressure, heart rate and temperature. Effect modifiers like age, duration of admission, gender was controlled by stratification. Post stratification chi-square test was applied and a $P$-value of less than .05 considered statistically significant.

\section{Results}

Out of 385 patients, 231 (60\%) were male while 154 (40\%) were females with maximum stay at hospital being 72 hours (Table I). Pseudomonas aeruginosa and Salmonella typhi were the most common bacterial organisms ( $n=83 ; 21.6 \%$ each) causing nosocomial blood stream infection (Figure 1).

Gender, an effect modifier was stratified and compared with frequency of most common bacterial organisms in nosocomial blood stream infections. There was predominance of Salmonella typhi and Pseudomonas aeruginosa in males while Mycobacterium tuberculosis was more frequent in females. Chi-square test was used to compare gender stratification with frequency of most common bacterial organisms in nosocomial blood stream infections which was statistically significant ( $P=.001)$ (Table II).

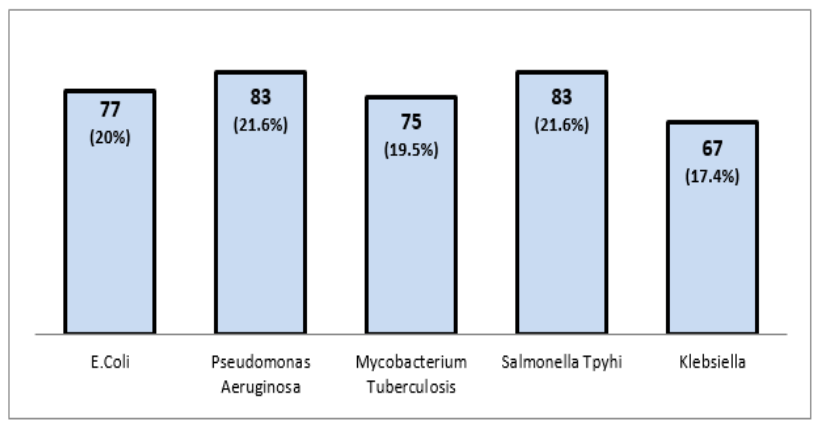

Figure 1: Frequency of bacterial organisms in nosocomial blood stream Infections

Similarly, effect modifiers like age and duration (hours) of admission to PICU were also compared with frequency of most common bacterial organisms in nosocomial blood stream infections. Each of the above effect modifiers were divided into two groups (Table II). Chi-square test was used to compare the frequency of different bacterial organisms between the two age groups as well as between two cut-off values for duration of stay in 
PICU. The results showed that there was no association between patients' age group and presence of different bacterial organisms. Similarly, there was no statistically significant difference between duration of admission to PICU and infection with different bacterial organisms $(P>.05)$ (Table II).

\begin{tabular}{|c|c|c|c|c|c|}
\hline \multicolumn{6}{|c|}{ Table: I Descriptive statistics of patients ( $n=385)$} \\
\hline \multicolumn{2}{|l|}{ Gender } & Male & $231(60 \%)$ & Female & $154(40 \%)$ \\
\hline \multicolumn{2}{|l|}{ Category } & Minimum & Maximum & Mean & Standard deviation \\
\hline \multicolumn{2}{|l|}{ Age } & 0.038 & 12 & 3.19 & 2.09 \\
\hline \multicolumn{2}{|c|}{ Duration of stay in PICU (hours) } & 48 & 72 & 55.85 & 8.38 \\
\hline \multirow{2}{*}{$\mathrm{BP}(\mathrm{mmHg})$} & Systolic & 109 & 125 & 119.17 & 2.8 \\
\hline & Diastolic & 41 & 80 & 58.11 & 10.29 \\
\hline \multicolumn{2}{|l|}{ Heart Rate } & 90 & 115 & 103.08 & 9.92 \\
\hline
\end{tabular}

\begin{tabular}{|c|c|c|c|c|c|}
\hline \multicolumn{6}{|c|}{ Table II: Comparison of effect modifiers with frequency of bacterial infection } \\
\hline \multirow[b]{2}{*}{ Gender } & \multicolumn{5}{|c|}{$\begin{array}{c}\text { Bacterial organisms } \\
\mathrm{n}(\%)\end{array}$} \\
\hline & E. coli & P. aeruginosa & M. tuberculosis & S. typhi & Klebsiella \\
\hline Male & $53(68.8)$ & $70(84.3)$ & $15(20.0)$ & $72(86.7)$ & $21(31.3)$ \\
\hline Female & $24(31.2)$ & $13(15.7)$ & $60(80.0)$ & $11(13.3)$ & $46(68.7)$ \\
\hline$P$-value ${ }^{*}$ & .077 & .001 & .001 & .001 & .001 \\
\hline \multicolumn{6}{|l|}{ Age groups } \\
\hline$\leq 6$ years & $73(94.8)$ & $76(91.6)$ & $70(93.3)$ & $79(95.2)$ & 65 (97) \\
\hline 7 - 12 years & $4(5.2)$ & $7(8.4)$ & $5(6.7)$ & $4(4.8)$ & $2(3.0)$ \\
\hline$P$-value ${ }^{*}$ & .826 & .228 & .228 & .692 & .29 \\
\hline \multicolumn{6}{|c|}{$\begin{array}{l}\text { Duration of Admission } \\
\text { to PICU (hours) }\end{array}$} \\
\hline $48-60$ hours & $58(75)$ & 68 (81.9) & $58(77.3)$ & $74(89.2)$ & 49 (73.1) \\
\hline $61-72$ hours & $19(24.7)$ & $15(18.1)$ & $17(22.7)$ & $9(10.8)$ & 18 (26.9) \\
\hline$P$-value ${ }^{*}$ & .281 & .576 & .576 & .563 & .139 \\
\hline
\end{tabular}

${ }^{*} P$-value $<.05$ was considered statistically significant

E. Coli-Escherichia coli; P. aeruginosa-Pseudomonas aeruginosa; M. tuberculosis-Mycobacterium tuberculosis; S. typhiSalmonella typhi

\section{Discussion}

Our study reports a high frequency of common bacterial organisms in nosocomial blood stream infection in children admitted to pediatric ICU, with a predominance of gram-negative bacteria including Pseudomonas aeruginosa and Salmonella typhi. Gender of the patients was an effect modifier with a significant relationship with frequency of most common bacterial organisms in nosocomial blood stream infection. Nosocomial infections (NI) are a common problem, especially in intensive care units (ICU). Morbidity and mortality are high in patients with nBSIs in both developing and developed countries. ${ }^{11,12}$ Failure of the immune system to contain infection at a focal site and consequent dissemination, are a major cause of high morbidity and mortality. ${ }^{13}$ The frequency of these infections, their epidemiology, and the invading organisms keep on changing along with evolving medical environment. The immunocompromised hospitalized patients who are often heavily dependent on medical support and indwelling devices are especially prone to these infections. ${ }^{14,15}$ 
A study conducted by Kasmi et al. showed an incidence of $22.8 \%$ of nosocomial infections with a rate of 30 per 1000 patients. According to this study, common $\mathrm{nBSI}$ included lower respiratory tract infection (10.5\%), with ventilator associated pneumonia (VAP) (6.8\%), catheter associated UTI (7.1\%) and blood stream infection (BSI) (4.2\%), with central line-associated BSI (CLABSI) (2.4\%). ${ }^{16}$ This study highlights the incidence of device associated nosocomial infections, while in our study frequency of common bacterial organisms causing nosocomial infection was observed based on blood culture reports.

Our study showed Gram-negative bacteria as the commonest micro-organisms involved in nBSI with predominance of Pseudomonas aeruginosa and Salmonella typhi. A study carried out in Egypt by ElSahrigy et al. showed Staphylococcus aureus, Acinetobacter and Klebsiella as the commonest microorganism among PICU patients. ${ }^{17}$ In this study common infections were respiratory tract infections due to which staphylococcus was the commonest microorganism, while our study focused on hospital acquired blood stream infections. Geographical difference may also be a reason for this variation in results.

In our study, the mean age was 3.19 years while a study conducted by Almuneef et al. from Riyadh Saudi Arabia, reported a mean age of 2.6 years. ${ }^{18}$ This difference in mean age between the two studies may be due to the reason that majority of the patients included in study by Almuneef et al. were suffering from congenital diseases, in which incidence of infections is usually high.

There were $60 \%$ male and $40 \%$ female patients in our study, whereas a study conducted in PICU of a tertiary care hospital of north India reported $76.92 \%$ male and $23.08 \%$ female patients. ${ }^{19}$ There is predominance of male gender in this study as well as in our study, however the difference between the two studies is difference in sample size. The Indian study included only 20 children with hospitalacquired infection out of a total of 119 admitted to PICU. ${ }^{19}$ An increase in sample size might affect the ratio of gender involvement.

In our study, the mean duration of admission to PICU was 55.85 hours, while Almuneef and colleagues found that the mean PICU stay was 30.20 days (720 hours) for catheter related BSI patients and 6.35 days (151 hours) for non-BSI patients $(P<0.0001) .{ }^{18}$ These prolonged hours of PICU stay in this study may be attributed to catheter insertion in all the enrolled patients, which was not the case in our study.

Blood pressure and heart rate of the patients were calculated and compared with age-specific review charts for early identification of clinical sepsis, as both these parameters are used as an important "pediatric severe sepsis screening tool". ${ }^{20}$

Our study highlights the current trends of bacterial organisms causing nBSI in PICU which would be helpful in timely administration of antibiotics against common pathogens, hence reducing morbidity and mortality.

\section{Conclusion}

There is a high frequency of common bacterial organisms in nosocomial blood stream infections in children, with a predominance of gram-negative bacteria including Pseudomonas aeruginosa and Salmonella typhi.

\section{Recommendations}

We recommend early identification of nosocomial infections in admitted patients, especially those with a hospital stay of more than 48 hours, so that morbidity and mortality rates due to blood stream infections can be minimized. 


\section{Acknowledgment}

We are thankful to all children and their parents who participated in our study.

\section{References}

1. Zingg W, Hopkins S, Gayet-Ageron A, Holmes A, Sharland $M$, Suetens $C$, et al. Health-care-associated infections in neonates, children, and adolescents: an analysis of paediatric data from the European Centre for Disease Prevention and Control point-prevalence survey. Lancet Infect Dis. 2017; 17(4): 381-9. Doi: 10.1016/S1473-3099(16)30517-5.

2. Tauhid SA, Chowdhury MA, Hoque MM, Kamal M, Haque E. Nosocomial Bloodstream Infections in Children in Intensive Care Unit: Organisms, Sources, Their Sensitivity Pattern and Outcome of Treatment. J Bangladesh Coll Physicians Surg. 2017; 35(3): 11522.

3. Haque A, Ahmed SA, Rafique Z, Abbas $Q$, Jurair $H$, Ali $S A$. Device-associated infections in a paediatric intensive care unit in Pakistan. J Hosp Infect. 2017; 95(1): 98-100.

4. Mitt $P$, Metsvaht $T$, Adamson $V$, Telling $K$, Naaber $P$, Lutsar I, et al. Five-year prospective surveillance of nosocomial bloodstream infections in an Estonian paediatric intensive care unit. J Hosp Infect. 2014; 86(2): 95-9. Doi: 10.1016/j.jhin.2013.11.002.

5. Jordan GI, Esteban TE, Bustinza AA, De Carlos VJC, García SP, Concha TJA, et al. Trends in nosocomial infections and multidrug-resistant microorganisms in Spanish pediatric intensive care units. Enferm Infecc Microbiol Clin. 2016; 34(5): 286-92. Doi: 10.1016/j.eimc.2015.07.010.

6. Ng YY, Abdel-Latif MEA, Gan CS, Siham A, Zainol H, Lum LCS. Impact of infection control training for interns on PICU-acquired bloodstream infections in a middle-income country. Singapore Med J. 2015; 56(9): 506-12.

7. Siddiqui B, Azmat R, Tikmani SS, Rafi S, Syed B, Khan $M T$, et al. Frequency of bloodstream infection in febrile neutropenic patients, experience from a developing country. Ann Med Surg. 2018; 34: 71-4.

8. Qureshi S, Fatima P, Mukhtar A, Zehra A, Qamar FN. Clinical profile and outcome of antibiotic lock therapy for bloodstream infections in pediatric hematology/oncology patients in a tertiary care hospital, Karachi, Pakistan. Int J Pediatr Adolesc Med. 2019; 6(1): 25-8.

9. Somroo T, Tikmani SS, Ali SA. Frequency and Etiology of Community-Acquired Bloodstream Infection in
Hospitalized Febrile Children. J Med Diagnostic Methods. 2016; 5(3).

10. Altun O, Botero-Kleiven S, Carlsson S, Ullberg M, Özenci V. Rapid identification of bacteria from positive blood culture bottles by MALDI-TOF MS following short-term incubation on solid media. J Med Microbiol. 2015 Nov 1; 64(11): 1346-52.

11. Obeiro CW, Seale AC, Berkley J. Empiric Treatment of Neonatal Sepsis in Developing Countries. Pediatr Infect Dis J. 2015; 34(6): 659-61. Doi: 10.1097/INF.0000000000000692

12. Riaz S, Jamal M. Surveillance of Hospital Acquired Infections in Newborn: A Challenge in Developing Countries. J Islamabad Med Dental Coll. 2016; 5(1): 59-62.

13. Denstaedt SJ, Singer BH, Standiford TJ. Sepsis and nosocomial infection: Patient characteristics, mechanisms, and modulation. Front Immunol. 2018; 9: 2446. Doi: 10.3389/fimmu.2018.02446

14. Esfahani BN, Basiri R, Mirhosseini SMM, Moghim S, Dolatkhah S. Nosocomial Infections in Intensive Care Unit: Pattern of Antibiotic-resistance in Iranian Community. Adv Biomed Res. 2017; 6.

15. Deng S, Feng S, Wang W, Zhu H, Gong Y. Bacterial Distribution and Risk Factors of Nosocomial Blood Stream Infection in Neurologic Patients in the Intensive Care Unit. Surg Infect. 2019 Jan 1; 20(1): 2530.

16. Kasmi I, Sallabanda S, Kasmi G. Nosocomial infections in a Pediatric Intensive Care Unit in Albania. Int J Sci Res. 2013; 5(1): 1570-6.

17. El-Sahrigy SAF, Shouman MG, Ibrahim HM, Abdel Rahman AMO, Habib SA, Khattab AA, et al. Prevalence and Anti-Microbial Susceptibility of Hospital Acquired Infections in Two Pediatric Intensive Care Units in Egypt. Maced J Med Sci. 2019; 7(11): 1744-9. Doi: org/10.3889/oamjms.2019.485

18. Almuneef MA, Memish ZA, Balkhy HH, Hijazi O, Cunningham $G$, Francis $C$. Rate, risk factors and outcomes of catheter-related bloodstream infection in a paediatric intensive care unit in Saudi Arabia. J Hosp Infect. 2006; 62(2): 207-13. Doi: 10.1016/j.jhin.2005.06.032

19. Sodhi J, Satpathy S, Sharma DK, Lodha R, Kapil A, Wadhwa $\mathrm{N}$, et al. Healthcare associated infections in paediatric intensive care unit of a tertiary care hospital in India: Hospital stay \& extra costs. Indian J Med Res. 2016 Apr 1; 143: 502-6.

20. Sepanski RJ, Godambe SA, Mangum CD, Bovat CS, Zaritsky AL, Shah SH. Designing a pediatric severe sepsis screening tool. Front Pediatr. 2014; 2: 56. Doi: 10.3389/fped.2014.00056 